\title{
Experimental evidence of the influence of organic compounds on pyrite morphologies
}

\author{
ARNAUD DUVERGER ${ }^{1,2}$, VINCENT BUSIGNY ${ }^{1}$, JEAN- \\ CHRISTOPHE VIENNET $^{2}$, JENNYFER MIOT ${ }^{2}$ AND \\ SYLVAIN BERNARD ${ }^{2}$ \\ ${ }^{1}$ Institut de Physique du Globe de Paris, Université de Paris \\ ${ }^{2}$ Institut de Minéralogie, de Physique des Matériaux et de \\ Cosmochimie, Sorbonne Université - CNRS - MNHN
}

Presenting Author: arnaud.duverger@normalesup.org

Pyrite $\left(\mathrm{FeS}_{2}\right)$ is abundantly found in modern and ancient sediments. It can be present in the form of framboid, a texture defined as "microscopic spheroidal to subspheroidal clusters of equidimensional and equimorphic microcrystals" [1]. Biogenic origin of framboidal pyrites fueled debates for decades and it is now generally foresaken due to the production of framboidal pyrites through purely abiotic laboratory syntheses. However, the presence of organic matter templates within pyrite spherules suggests a potential contribution of microorganisms to the formation of these pseudo-framboids.

In order to explore further criteria of biogenic pyrite identification, we performed experimental diagenesis on both abiotic and biogenic iron-sulfur-phosphate systems. Biogenic starting materials were formed from a pure culture of Desulfovibrio desulfuricans supplied with nanoparticulate iron phosphate as an iron source. Abiotic starting material were produced from a mixture of the same iron phosphate source and abiotically precipitated iron sulfide. Experimental simulations were performed in hydrothermal reactors maintained at $75{ }^{\circ} \mathrm{C}$ or $150{ }^{\circ} \mathrm{C}$ for 1 to 10 days. Products were characterized by X-ray diffraction and scanning and transmission electron microscopies.

Experimental diagenesis led to a mixture of pyrite and lipscombite-like phosphate $\left(\mathrm{Fe}^{\mathrm{II}}{ }_{\mathrm{x}} \mathrm{Fe}^{\mathrm{III}}{ }_{(3-\mathrm{x})}\left(\mathrm{PO}_{4}\right)_{2}(\mathrm{OH})_{(3-\mathrm{x})}\right)$ from both abiotic and biogenic starting materials. While abiotic starting materials led to the formation of submicrometric single crystals of pyrite with euhedral shapes similar to the subunits of well crystallized framboids, biogenic starting materials produced micrometric crumpled spherules of pyrite resembling the so-called pseudo-framboids. Although additional experiments appear required to go further, such specific morphology, likely related to the presence of organic materials, may help recognizing 'biogenic' pyrite in the geological record.

[1] Ohfuji \& Rickard (2005). Earth-Science Reviews 71, $147-170$. 